OPEN ACCESS

Edited by:

Hyun Park

National Cancer Institute (NCl),

United States

Reviewed by:

Vibhuti Dave,

Hôpital Maisonneuve-Rosemont,

Canada

Qi-Jing Li,

Duke University, United States

*Correspondence:

Helena Nunes-Cabaço

hcabaco@medicina.ulisboa.pt

Ana E. Sousa

asousa@medicina.ulisboa.pt

${ }^{\dagger}$ Present address:

Leila R. Martins,

Division of Applied Functional

Genomics, German Cancer Research Center (DKFZ) and National Center for

Tumor Diseases (NCT) Heidelberg,

Heidelberg, Germany

Specialty section:

This article was submitted to

T Cell Biology,

a section of the journal

Frontiers in Immunology

Received: 12 December 2021

Accepted: 24 January 2022

Published: 11 February 2022

Citation:

Nunes-Cabaço $\mathrm{H}$,

Ramalho-dos-Santos $A$,

Pires AR, Martins LR,

Barata JT and Sousa AE

(2022) Human CD4 T Cells

From Thymus and Cord Blood

Are Convertible Into CD8

$T$ Cells by IL-4.

Front. Immunol. 13:834033. doi: 10.3389/fimmu.2022.834033

\section{Human CD4 T Cells From Thymus and Cord Blood Are Convertible Into CD8 T Cells by IL-4}

\author{
Helena Nunes-Cabaço*, Andreia Ramalho-dos-Santos, Ana R. Pires, Leila R. Martins ${ }^{\dagger}$, \\ João T. Barata and Ana E. Sousa*
}

Instituto de Medicina Molecular João Lobo Antunes, Faculdade de Medicina, Universidade de Lisboa, Lisbon, Portugal

Commitment to the CD4+ or CD8+ T cell lineages is linked to the acquisition of a functional program broadly defined by helper and cytotoxic properties, respectively. The mechanisms underlying these processes in the human thymus remain largely unclear. Moreover, recent thymic emigrants are thought to have some degree of plasticity, which may be important for the shaping of the immune system and adjustment to specific peripheral needs. We show here that IL-4 induces proliferation-independent de novo synthesis of CD8 $\alpha \beta$ in human CD4 single-positive (SP) thymocytes, generating a stable CD8SP population that features a diverse TCR $\alpha \beta$ repertoire, CD4 expression shut-down and ThPOK downregulation. IL-4 also promotes an innate-like program in both CD4SP and CD8SP thymocytes, characterized by Eomes upregulation in the absence of T-bet, in line with its recognized role in the generation of thymic innate-like CD8+ T cells. The clinical relevance of these findings is further supported by the profile of IL-4 production and IL-4 receptor expression that we identified in the human thymus. Importantly, human cord blood CD4+ T cells preserve the ability to generate Eomes+ CD8+ T cells in the presence of IL-4, with implications in neonatal immunity. Our results support a role for IL-4 in the dynamic regulation of human thymocyte plasticity and identify novel strategies to modulate immune responses.

Keywords: CD4+ and CD8+ T cell lineage commitment, IL-4, human thymus, cord blood, innate-like T cells

\section{INTRODUCTION}

$\mathrm{CD}^{+}$and $\mathrm{CD}^{+} \mathrm{T}$ cell development follow a stringent program in the thymus that ensures highly efficient peripheral responses. This involves validation of TCR specificity together with cytokine signaling, leading to the formation of discrete helper or cytotoxic lineages, respectively. However, many of the mechanisms underlying lineage commitment remain unclear, as well as the degree of cell plasticity. In humans, hematopoietic precursors first acquire CD4 (CD4 immature single positive thymocytes, CD4ISP) and then $\mathrm{CD} 8$ expression, generating $\mathrm{CD} 4^{+} \mathrm{CD} 8^{+}$double positive (DP) cells that will undergo lineage commitment into helper CD4 single-positive (CD4SP) or cytotoxic CD8SP thymocytes (1). Thymocyte maturation has been discretely classified in terms of the expression of CD69, CD27, CD1a and CD45RA (1-3). Positive selection is associated with CD69 up-regulation at the DP $\mathrm{CD} 3^{\mathrm{dim}}$ to $\mathrm{CD} 3^{\text {high }}$ stages in $\mathrm{CD} 1 \mathrm{a}$-expressing thymocytes. Subsequent 
CD27 acquisition has been linked to lineage commitment, since DPs expressing CD27 can only differentiate into CD8SP in fetal thymic organ cultures (FTOC), while expression of CD27 on CD4SP thymocytes associates with commitment to the CD4 lineage. Finally, downregulation of CD1a and expression of CD45RA on SP cells mark acquisition of a mature phenotype and potential for thymic egress (1-3).

Mutual antagonistic repression of the transcription factors ThPOK (T-helper-inducing POZ/Krüppel-like factor; also known as cKrox and encoded by the $Z b t b 7 b$ gene, hereafter referred to as ThPOK) and the Runx3 member of the Runx family dictates lineage fate in the thymus, as observed in mouse models $(4,5)$. ThPOK is a crucial regulator of CD4 lineage development that is necessary and sufficient for helper $\mathrm{T}$ cell commitment, directing positively selected thymocytes to a CD4 fate regardless of the MHC specificity of their TCR (4-7). Conversely, Runx3 promotes CD8 lineage commitment, namely through $C d 4$ silencing $(5,8,9)$. In addition, the zinc-finger transcription factor GATA-3 is induced by TCR signal strength and its requirement appears to precede ThPOK in CD4 $\mathrm{T}$ cell development $(10,11)$. Another transcription factor termed Mazr (Myc-associated zinc finger-related factor) has been shown to contribute to CD8 lineage commitment through repression of ThPOK expression, although it may also regulate CD4 commitment (12).

Once the helper vs. cytotoxic lineage fate is established, mature SP thymocytes shut down the expression of the other coreceptor. The CD8-CD4 lineage dichotomy persists in peripheral mature T cells, as ThPOK continues to suppress the cytotoxic fate in CD4 T cells (13). Nevertheless, some degree of plasticity is still evident in CD4 T cells upon chronic or strong stimulation, suggesting that CD8 repression may be reversible in particular microenvironments, as reported in the gut mucosa $(14,15)$.

Studies in mice have established a kinetic lineage commitment model whereby developing TCR $\alpha \beta^{+} \mathrm{CD} 4^{+} \mathrm{CD} 8^{+} \mathrm{DP}$ thymocytes undergoing positive selection initially terminate $\mathrm{CD} 8$ transcription in order to test their TCR specificity. At this point, a long and persistent TCR signaling leads to CD4SP cell commitment, whereas rapid loss of TCR engagement together with suitable cytokine signaling result in reactivation of $C d 8$ expression and cytotoxic CD8SP cell generation $(5,16)$. IL-7 has been shown to be the main cytokine involved in this process, termed "coreceptor reversal", although the involvement of other $\gamma$-chain $(\gamma c)$ cytokines has also been reported (17).

Interleukin-4 (IL-4), a short four-helix bundle peptide member of the common $\gamma c$ receptor cytokine family, is a pleiotropic cytokine produced mainly by lymphocytes, namely Th2, $\gamma \delta \mathrm{T}$, NKT and T follicular helper cells, as well as by mast cells, basophils and eosinophils (18). IL-4 regulates different processes in multiple cell types. In addition to its role in B cell differentiation, it promotes Th2 by inhibiting Th1 and Th17 fates in T cells (19). IL-4 is also involved in CD8 T cell function, as it promotes the proliferation and cytotoxic activity of CD8 $\mathrm{T}$ cells $(20-22)$ or can act as a negative regulator of CD8 $\mathrm{T}$ cell responses (23). In the thymus, IL-4 is required for the development of innate-like CD8 $\mathrm{T}$ cells, which feature phenotypical and functional traits of memory $\mathrm{T}$ cells in the absence of foreign antigen stimulation (24). Innate-like CD8 T cells express high levels of CD44, CXCR3, CD122 and Eomes but not T-bet, and are rapid IFN- $\gamma$ producers upon activation (24). Their development is highly dependent on IL-4 production by cells expressing the promyelocytic leukemia zinc finger protein (PLZF), which include invariant NKT (iNKT) cells in mice and CD4 $\mathrm{T}$ cells that resulted from MHC class II-dependent thymocyte-thymocyte interactions (T-T CD4) in humans (25). Eomes ${ }^{+}$innate-like CD8 $\mathrm{T}$ cells were identified in human fetal thymuses and in cord blood, but their thymic expression was reported to rapidly decrease after birth until 3 months of age (26, 27). Importantly, responsiveness to IL-4 during murine CD8 T cell development and homeostasis may alter their functional reactivity and response to pathogens (28).

Here, we found that IL- 4 upregulates CD $8 \alpha \beta$ and downregulates CD4 and ThPOK in human CD4SP thymocytes, leading to their conversion into CD8 T cells. Moreover, a population of innate-like cells is induced, as defined by the expression of Eomes and CXCR3 in the absence of T-bet. The effects of IL-4 extended to CD4 T cells from cord blood and progressively declined in the periphery with age and cell differentiation stage, indicating a preferential impact in the neonatal period.

\section{MATERIALS AND METHODS}

\section{Human Samples}

Human thymic specimens were from newborns to 14-month-old children undergoing routine thymectomy performed during corrective cardiac surgery at Hospital de Santa Cruz, Carnaxide, Portugal. Cord blood was collected at the Department of Obstetrics of Hospital de Santa Maria/Centro Hospitalar Universitário Lisboa Norte (CHULN), Lisbon, Portugal. Pediatric tonsils and paired blood were from 4- to 5year-old children and were obtained from routine pediatric tonsillectomy at the ENT department of Hospital de Santa Maria/CHULN, Lisbon, Portugal. All samples were collected after parent's/tutor's written informed consent. The study was approved by the Ethical Boards of Hospital de Santa Cruz, Faculty of Medicine of the University of Lisbon and CHULN.

\section{Sample Processing}

Total thymocytes and tonsillar cells were recovered through tissue dispersion using a $70 \mu \mathrm{M}$ filter and a syringe plunger. Mononuclear cells were separated from tissue suspension or from heparinized cord, pediatric or adult blood using FicollPaque PLUS (GE Healthcare) density gradient. The cord blood mononuclear cell suspension was further incubated with BD FACS Lysing Solution (BD Biosciences) for red blood cell lysis.

\section{Cell Separation, Labeling and Culture}

CD4SP or CD8SP cells were sorted (purity $>98 \%$ ) from total thymocytes as $\mathrm{CD} 3^{\text {high }} \mathrm{CD} 4^{+} \mathrm{CD} 8^{\text {neg }} \mathrm{CD} 25^{\text {neg }} \mathrm{TCR} \gamma \delta^{\text {neg }}$ or $\mathrm{CD} 3{ }^{\text {high }} \mathrm{CD} 4^{\text {neg }} \mathrm{CD} 8^{+} \mathrm{CD} 25^{\text {neg }} \mathrm{TCR} \gamma \delta^{\text {neg }}$ cells, respectively, using 
a FACSAria High-Speed Cell Sorter (BD Biosciences). CD4+ T cells were FACS-purified from blood or tonsils as $\mathrm{CD} 3^{\text {high }} \mathrm{CD}^{+} \mathrm{CD} 8^{\text {neg }}$ (purity $>97 \%$ ). Anti-human monoclonal antibodies (mAbs) used were: CD3 (UCHT1), CD4 (RPA-T4), $\mathrm{CD} 8 \alpha$ (RPA-T8), CD25 (2A3) and TCR $\gamma \delta$ (B1.1) from eBioscience/Thermo Fisher Scientific or BD Biosciences. Where indicated, cells were labeled with $1 \mu \mathrm{M}$ CellTrace Violet (CTV; Thermo Fisher Scientific) for 20 minutes at room temperature. Sorted populations were resuspended at $1-2 \times 10^{6}$ cells/mL in complete medium (RPMI1640 with $10 \%$ fetal bovine serum (FBS), 2mM L-glutamine and $50 \mathrm{~m} / \mathrm{mL}$ penicillin/ streptomycin, all from GIBCO/Invitrogen) and cultured in the presence or absence of IL-2 (20U/mL), IL-4 (20ng/mL; R\&D or Peprotech) or IL-7 (10ng/mL, R\&D) for 6 or 7 days at $37^{\circ} \mathrm{C}$ and $5 \% \mathrm{CO}_{2}$. Where mentioned, an anti-IL-4 neutralizing antibody (MP4-25D2, eBioscience) was added to the IL-4 culture at the specified concentrations. For gene expression analysis, thymocyte populations were FACS-sorted as TN $\left(\mathrm{Lin}^{\text {neg }} \mathrm{CD} 3^{\text {neg }}\right.$ $\left.\mathrm{CD} 4{ }^{\text {neg }} \mathrm{CD} 8^{\text {neg }} \mathrm{CD} 34^{+}\right)$, CD4ISP $\left(\mathrm{Lin}^{\text {neg }} \mathrm{CD} 3^{\text {neg }} \mathrm{CD} 4^{+} \mathrm{CD} 8^{\text {neg }}\right)$, $\mathrm{DP}\left(\mathrm{Lin}^{\text {neg }} \mathrm{CD} 4^{+} \mathrm{CD} 8^{+}\right), \mathrm{CD} 4 \mathrm{SP}\left(\mathrm{Lin}^{\text {neg }} \mathrm{CD} 3{ }^{\text {high }} \mathrm{CD} 4^{+} \mathrm{CD} 8^{\text {neg }}\right)$ or CD8SP ( Lin $^{\text {neg }} \mathrm{CD} 3{ }^{\text {high }} \mathrm{CD} 4{ }^{\text {neg }} \mathrm{CD} 8^{+}$) cells using additional antihuman mAbs such as CD11c (3.9), CD14 (61D3), CD16 (eBioCB16), CD19 (HIB19), CD20 (2H7), CD34 and CD123 (6H6) from eBioscience/Thermo Fisher Scientific. Sorted cells were frozen as dry pellets and stored at $-80^{\circ} \mathrm{C}$ until further use.

\section{Flow Cytometry Staining and Analysis}

Surface staining was performed for 20 minutes at room temperature and always included Fixable Viability Dye (eBioscience) for dead cell exclusion. For intracellular analysis thymocytes were fixed, permeabilized and stained using the Transcription Factor Staining Buffer Set (eBioscience), as per manufacturer's instructions. Other anti-human mAbs used included: CD8 $\beta$ (SIDI8BEE), CD27 (O323), CD45RA (HI100), CD44 (IM7), CD69 (FN50), CXCR3 (1C6), Eomes (WD1928), T-bet (eBio4B10), ThPOK (ZFP-67), Runx1 (RXDMC), Runx3 (R3-5G4) and Ki67 (B56) from eBioscience/Thermo Fisher Scientific, BD Biosciences or R\&D Systems. Cells were acquired in a LSRFortessa (BD Biosciences) cytometer and data was analyzed using FlowJo v10 (FlowJo, BD). For the visualization of high-dimensional data through the $t$ distributed stochastic neighbor embedding ( $t$-SNE) dimensionality reduction technique, similar number of live, single cells from each condition were concatenated. tSNE parameters were set to 1,000 iterations, perplexity 30 and learning rate 4200 , and based on appropriate markers.

\section{Thymic Organ Cultures (TOCs)}

Thymic tissue blocks (1-2 mm diameter) were placed on Isopore membranes (Millipore) in a 6 well plate containing $2 \mathrm{~mL}$ of TOC medium (complete medium but with 15\% FBS and addition of $10 \mathrm{mM}$ HEPES, $1 \mathrm{mM}$ sodium pyruvate and 1\% MEM nonessential aminoacids, all from GIBCO/Invitrogen), without (control) or with addition of IL-4 $(10 \mathrm{ng} / \mathrm{ml})$, and cultured at $37^{\circ} \mathrm{C}$ and $5 \% \mathrm{CO}_{2}$. A third of the media was replaced every 2-3 days. On day 6 post-infection TOCs were mashed and thymocytes were stained and analyzed by flow cytometry.

\section{Functional Studies}

CD4SP thymocytes were cultured for 7 days with IL-4 and subsequently FASC-sorted into tCD4SP $\left(\mathrm{CD} 4^{+} \mathrm{CD} 8^{\text {neg }}\right)$, iDP $\left(\mathrm{CD} 4^{+} \mathrm{CD}^{+}\right)$and $\mathrm{iCD} 8 \mathrm{SP}\left(\mathrm{CD} 4^{\text {neg }} \mathrm{CD} 8^{+}\right)$populations. Sorted cells were either stimulated with 50ng/ml PMA and 500ng/ml ionomycin in the presence of $10 \mu \mathrm{g} / \mathrm{mL}$ brefeldin for $4 \mathrm{~h}$ at $37^{\circ} \mathrm{C}$ and $5 \% \mathrm{CO}_{2}$, or they were further TCR-stimulated using Dynabeads Human T-activator CD3/CD28 (Thermo Fisher Scientific) for 5 days before the PMA/ionomycin protocol. The same stimulation procedures were applied to ex vivo CD4SP and CD8SP cells, as controls. After stimulation cells were surfacestained, fixed with formaldehyde $2 \%$, permeabilized with $0,5 \%$ saponin in PBS/BSA/azide and incubated with intracellular antibodies, including anti-IFN- $\gamma$ (4S.B3) and anti-TNF- $\alpha$ (MAb11) from Thermo Fisher Scientific, before acquisition in an LSRFortessa cytometer.

\section{IL-4 Production by Thymocytes Ex Vivo}

Total thymocytes were incubated with $10 \mu \mathrm{g} / \mathrm{mL}$ brefeldin for $4 \mathrm{~h}$ at $37^{\circ} \mathrm{C}$ and $5 \% \mathrm{CO}_{2}$, surface-stained, fixed with formaldehyde $2 \%$ and permeabilized with $0,5 \%$ saponin before incubation with intracellular antibodies. Anti-human mAbs used included: CD16 (eBioCB16), CD56 (NCAM), HLA-DR (L243) and anti-IL-4 (8D48), all from Thermo Fisher Scientific, Biolegend or BD Biosciences. The following live $\left(\mathrm{FVD}^{\text {neg }}\right)$, singlet thymocyte populations were analyzed: $\gamma \delta \mathrm{T}$ cells $\left(\mathrm{CD} 3^{\text {high }} \mathrm{TCR} \gamma \delta^{+}\right)$; $\mathrm{CD} 16^{+} / \mathrm{CD}^{+} 6^{+}$cells $\left(\mathrm{TCR} \gamma \delta^{\text {neg }} \mathrm{CD} 16^{+} / \mathrm{CD} 56^{+}\right)$; and within $\mathrm{TCR} \gamma \delta^{\text {neg }} \mathrm{CD} 16^{\text {neg }}$ CD $56^{\text {neg }}$ cells: TN (CD $\left.3^{\text {neg }} C D 4^{\text {neg }} C D 8^{\text {neg }}\right), C D 4 I S P$ $\left(\mathrm{CD} 3^{\text {neg }} \mathrm{CD} 4^{+} \mathrm{CD} 8^{\text {neg }}\right)$, DP $\mathrm{CD} 3^{\text {low }}\left(\mathrm{CD} 3^{\text {low }} \mathrm{CD} 4^{+} \mathrm{CD} 8^{+}\right), \mathrm{DP}$ $\mathrm{CD} 3^{\text {high }}\left(\mathrm{CD} 3^{\text {high }} \mathrm{CD} 4^{+} \mathrm{CD} 8^{+}\right), \mathrm{CD} 4 \mathrm{SP}\left(\mathrm{CD} 3^{\text {high }} \mathrm{CD} 4^{+} \mathrm{CD} 8^{\text {neg }}\right)$, CD8SP $\left(\mathrm{CD} 3^{\text {high }} \mathrm{CD} 4^{\text {neg }} \mathrm{CD} 8^{+}\right)$and $\mathrm{HLA}-\mathrm{DR}^{+}$cells.

\section{Gene Expression Quantification}

$100 \mathrm{ng}$ of total RNA purified with ZR-Duet DNA/RNA MiniPrep Kit (Zymo Research) were used to synthesize cDNA using random primers and Superscript III (Invitrogen). For the quantification of IL4RA and ACTB (reference gene), cDNA pre-amplification and real-time PCR (with TaqMan ${ }^{\circledR}$ PreAmp and Taqman ${ }^{\circledR}$ Gene Expression Master Mix, respectively; Applied Biosystems) were performed using gene expression assays (IL4RA: Hs00166237_m1; ACTB: Hs99999903_m1; Thermo Fisher Scientific). EOMES, TBX21 (T-bet), MAZR, ETS1 and IL13RA were quantified using predesigned KiCqStart ${ }^{\circledR}$ SYBR ${ }^{\circledR}$ Green Primers (Sigma-Aldrich) and Power SYBR ${ }^{\circledR}$ Green Master Mix (Thermo Fisher Scientific). Quantification of CD4, CD8, ThPOK (ZBTB7B), GATA3, RUNX1, RUNX2, RUNX3 and $A C T B$ was also performed with $S Y B R^{\circledR}$ Green using Applied Biosystems ViiA7 Real-Time PCR System, and the following primers were used (Forward/Reverse): CD4: TGCCTCAGTATGCTGGCTCT/GAGACCTTTGCCT CCTTGTTC; CD8: TCCTCCTATACCTCTCCCAAAAC/GG AAGACCGGCACGAAGTG; ZBTB7B: GTCCCCAGAG CTACGAACC/AGCTTAGGTAGGCCATCAGGT; GATA3: GCCCCTCATTAAGCCCAAG/TTGTGGTGGTCTGA CAGTTCG; RUNX1: ACTATCCAGGCGCCTTCACCTACT/ T A G T A C A G G T G G T A G GA G G G C GA G; RUNX2: ACGAATGCACTATCCAGCCACCTT/ATATGGAGTGCTGC TGGTCTGGAA; RUNX3: AGGCAATGACGAGAACTACTCC/ 
CGAAGGTCGTTGAACCTGG; ACTB: CTGGCACCCAG CACAATG/GCCGATCCACACGGAGTACT.

\section{Immunohistochemistry}

Formalin-fixed thymic tissue was embedded in paraffin and cut into $3 \mu \mathrm{m}$ sections (Minot Microtome Leica RM2145). Epitoperetrieval was performed at $\mathrm{pH} 9$ (Leica Biosystems buffer) for $15 \mathrm{~min}$ using a microwave $(800 \mathrm{~W})$. Samples were stained with antibodies against human IL-4, incubated with a peroxidase/ DAB detection system (EnVision, Dako) and counterstained with Harris' hematoxylin (BioOptica). Images were acquired using a Leica DM2500 microscope.

\section{Mouse Studies}

CD4SP cells were sorted from the mashed thymus of 9-day-old or 5-week-old C57BL/6 mice as TCR $\beta^{\text {high }} \mathrm{CD} 4^{+} \mathrm{CD} 8^{\text {neg }}$ (purity $>98 \%$ ) using a BD FACSAria High-Speed Cell Sorter, cultured with murine IL-4 (20 ng/ml; Peprotech) or IL-7 (10 ng/ml) for 6 days as described above and analyzed by flow cytometry.

\section{Statistics}

Statistical analysis was performed using GraphPad Prism v7 (GraphPad Software Inc.) and results are presented as mean \pm SD. Two-sample data were compared using Wilcoxon-matched pairs test. Data from more than two samples were compared using Friedman test or Kruskal-Wallis test with Dunn's multiple comparison post-test, considering CD4SP thymocytes ex vivo as control values whenever cells were cultured with cytokines. Only statistically significant data $(\mathrm{P}$ value $<0.05)$ is presented.

\section{RESULTS}

\section{IL-4 Induces the Generation of Mature DP and CD8SP Thymocytes From Human CD4SP Thymocytes}

We noticed a marked upregulation of $\mathrm{CD} 8 \alpha \beta$ on human CD4SP thymocytes when cultured in the presence of IL-2, IL-4 and IL-7 to facilitate cell survival in studies of HIV infection (29). This prompted us to investigate which cytokine was responsible for this effect by culturing CD4SP thymocytes, depleted of CD25 ${ }^{+}$ and TCR $\gamma \delta^{+}$cells, in the presence of each cytokine (Figure 1A). IL-4 displayed the strongest ability to promote CD $8 \alpha \beta$ expression, resulting in the generation of induced double positive DP (iDP) and CD8SP (iCD8SP) cells (Figures 1A, B and Figure S1A), irrespective of the concentration used (Figure S1B; above $10 \mathrm{ng} / \mathrm{ml}$ for iDP cells), the time-point analyzed (Figure S1C) or the age of the thymic donor (Figure S1D). Both iDP and iCD8SP cells maintained high CD3 levels, in agreement with preservation of a mature phenotype (Figure S1E). In addition, neither IL-13 nor IL-15, whose receptors share IL$4 \mathrm{R} \alpha$ and $\gamma \mathrm{c}$ subunits with the IL-4 receptor, respectively, were able to induce CD8 expression on CD4SP thymocytes (Figure S1F). Notably, addition of IL-4 to thymic organ cultures (TOCs) markedly reduced the CD4SP/CD8SP ratio (Figure S1G), supporting its impact also in the tissue microenvironment.
Moreover, the addition of an anti-IL-4 neutralizing antibody to the IL-4 culture decreased the upregulation of CD8 and the generation of iCD8SP cells (Figure S1H).

Importantly, the increase in $\mathrm{CD} 8 \alpha$ protein in iCD8SP cells was paralleled by $C D 8 A$ upregulation and the shutdown of $C D 4$ at the transcriptional level (Figure 1C). CD8A upregulation was already noticeable at $4 \mathrm{~h}$ post-IL- 4 culture (mean \pm SD foldchange $1,9 \pm 0,6, \mathrm{n}=3$ ). While $\mathrm{CD} 4$ is a monomeric type I transmembrane glycoprotein, CD8 exists as two isoforms, $\mathrm{CD} 8 \alpha \alpha$ and $\mathrm{CD} 8 \alpha \beta$, expressed on different cell types and with different functions. Conventional CD8SP thymocytes express $\mathrm{CD} 8 \alpha \beta$, which has been associated with greater signaling ability than CD8 $\alpha \alpha$ (30). Mice lacking $C D 8 \beta$ are devoid of peripheral mature $\mathrm{CD} 8 \mathrm{~T}$ cells (31). Thus, we examined the composition of the CD8 molecule upregulated in CD4SP thymocytes after IL- 4 exposure. Indeed, we confirmed that iDP and iCD8SP expressed the CD8 $\beta$ subunit, and thus consisted of the $C D 8 \alpha \beta$ heterodimer (Figure 1D and Figure S1E).

Interestingly, IL-4 was able to induce CD8 expression in both $\mathrm{CD} 27^{\text {neg }}$ and $\mathrm{CD} 27^{+} \mathrm{CD} 4 \mathrm{SP}$ thymocytes (Figure S1I). Moreover, although the potential to respond to IL-4 declined with CD4SP maturation, as assessed by the expression of CD27 and CD45RA, we observed the generation of iCD8SP cells even from $\mathrm{CD} 27^{+} \mathrm{CD}_{45 \mathrm{RA}^{+}}$thymocytes (Figure S1J).

We then asked whether IL-4 was also able to induce CD8 expression on murine CD4SP thymocytes in vitro. In comparison to the human thymus, we found very low CD8 upregulation and reduced generation of iDP and iCD8SP cells upon exposure of mouse TCR $\beta^{\text {high }}$ CD4SP thymocytes to IL-4 (Figure S1K). Of note, in the murine system equivalent to the human system used both IL-4 and IL-7 had low ability to induce CD8 expression on CD4SP thymocytes (Figure S1K).

Next, we investigated the ability of IL-4 to upregulate CD8 expression on peripheral CD4 $\mathrm{T}$ cells at different ages and differentiation stages, including: i) total $\mathrm{CD} 4 \mathrm{~T}$ cells from cord blood; ii) naïve or memory CD4 $\mathrm{T}$ cells from tonsil or paired pediatric blood; and iii) naïve or memory $\mathrm{CD} 4 \mathrm{~T}$ cells from adult blood. Cord blood CD4 T cells displayed the highest ability to increase $\mathrm{CD} 8 \alpha \beta$ expression and to generate $\mathrm{CD} 8 \alpha \beta^{+} \mathrm{CD} 4^{\text {neg }} \mathrm{T}$ cells upon exposure to IL-4 (Figures 1E, F). Moreover, the potential for IL-4-induced CD8 $\alpha \beta$ upregulation decreased both with age (cord blood $>$ pediatric blood $>$ adult blood), and differentiation stage (naïve>memory), with memory CD4 T cells of any source showing a negligible capacity to increase CD8 expression upon IL-4 culture (Figures 1E, F).

In conclusion, we found that IL-4 was able to induce coreceptor reversal in human CD4SP thymocytes, generating iDP and iCD8SP thymocytes with a mature phenotype. Importantly, our observation that IL-4 induced the generation of $\mathrm{CD} 8 \alpha \beta^{+} \mathrm{CD} 4^{\text {neg }} \mathrm{T}$ cells from cord blood CD4 T cells extends the results obtained in the thymus to the periphery.

\section{CD4SP Thymocytes Express IL-4R $\alpha$ and Produce High Levels of IL-4}

The strong direct effect of IL-4 on CD4SP thymocytes indicates that this subset expresses the IL-4 receptor. Indeed, the ex vivo analysis of different thymocyte subsets revealed that CD4SP 

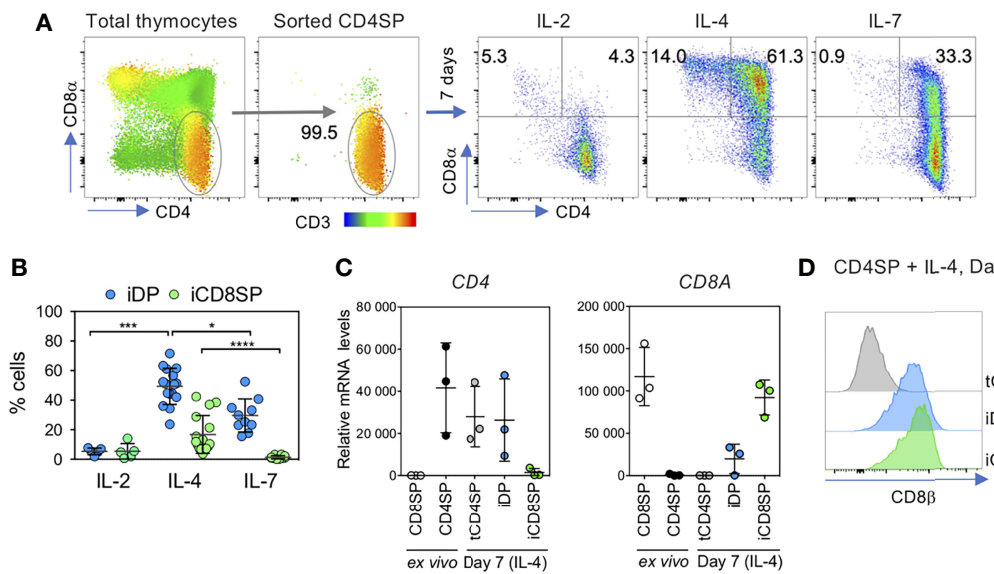

D $\quad$ CD4SP + IL-4, Day 7
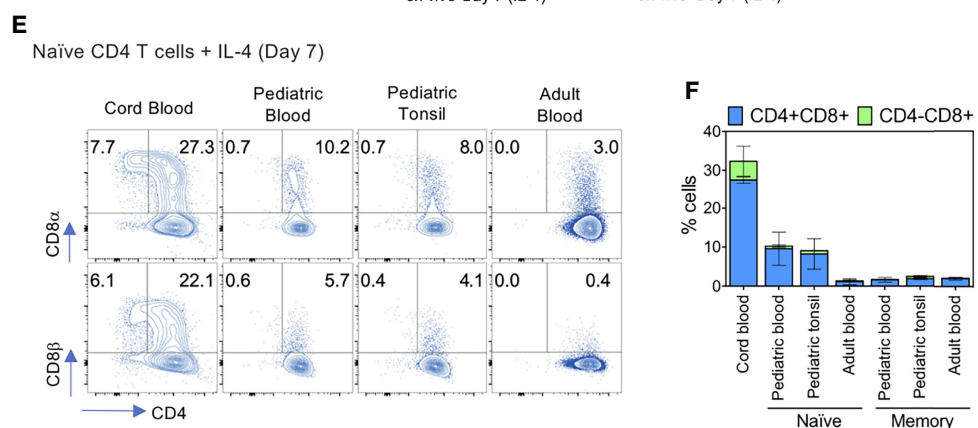

FIGURE 1 | IL-4 induces the upregulation of CD8 $\alpha \beta$ on human CD4SP thymocytes and in peripheral CD4 ${ }^{+} \mathrm{T}$ cells. (A) CD4SP thymocytes, sorted as $\mathrm{CD} 3^{\text {high }} \mathrm{CD}^{+}{ }^{+} \mathrm{CD} 8^{\text {neg }} \mathrm{CD} 25^{\text {neg }} \mathrm{TCR} \gamma \delta^{\text {neg }}$, were incubated with IL-2, IL-4 or IL-7 for 7 days and CD8 $\alpha$ expression was assessed at the end of the culture. CD3 expression is presented as an heatmap overlay on initial populations. (B) Frequency of cytokine-induced double-positive (iDP) and CD8 single-positive (iCD8SP) thymocytes after 7 days of culture with IL-2, IL-4 or IL-7. Each dot represents a thymus. Statistics was only performed within the same subset (iDP or iCD8SP). (C) CD4 and CD8A mRNA expression in CD4SP and CD8SP thymocytes ex vivo and in sorted populations 7 days after IL-4 culture. tCD4SP: cytokine-treated CD4 single-positive thymocytes. (D) Expression of CD8ß on tCD4SP, iDP and iCD8SP generated from incubation of CD4SP thymocytes with IL-4 for 7 days. (E) Expression of CD8 $\alpha$ and CD8 $\beta$ on sorted naïv $C D 4^{+} T$ cells from cord, pediatric or adult blood and pediatric tonsil after a 7-day culture with IL-4 (left dot plots) and $\mathbf{( F )}$ quantification of iDP and iCD8SP cells generated from naïve and memory T cells (right panel; $n=2 / 3$ ). Results in graphs are presented as mean $\pm S D$. ${ }^{*} p<$ $0.05,{ }^{\star \star \star} \mathrm{p}<0.001,{ }^{\star \star \star \star *} \mathrm{p}<0.0001$.

thymocytes expressed high levels of IL4RA mRNA, comparable to the expression observed on $\gamma \delta$ T cells (Figure 2A). Of note, we could not detect IL13RA mRNA expression in CD4SP thymocytes, in agreement with the lack of an effect of IL-13 on these cells.

Moreover, in support of a physiological role of IL-4 on CD4SP thymocytes, we found its expression in the human thymus, mainly in the medulla, by immunohistochemistry (Figure 2B). Flow cytometry analysis confirmed the existence of a clear population of IL-4-producing thymocytes, even in the absence of stimulation (mean \pm SD $5.7 \pm 2.4 \%$ of total thymocytes, $n=3$; Figure $2 \mathrm{C}$ and Figure S2A). Although the largest contributors to the IL-4 pool were DP CD $3^{\text {low }}$ cells, the amount of cytokine on a per cell basis in this population was relatively low (Figure 2D). Interestingly, CD4SP thymocytes not only represented a high proportion of the IL-4-producing thymocytes, but also featured the highest levels of IL-4 production at the single-cell level (Figures 2C, D). Of note, we found sustained thymic IL-4 production up to 14 months of age, the age of the oldest child submitted to reconstructive cardiac surgery during the study period (Figure S2B).
Overall, we showed that CD4SP thymocytes express high levels of IL- $4 \mathrm{R} \alpha$ and that IL- 4 is mainly produced in the thymic medulla by several types of cells after cells, including CD4SP thymocytes themselves. Our data thus support a possible physiological role of IL-4 in human CD8SP thymocyte development from CD4SP thymocytes.

\section{iCD8SP Thymocytes Are Stable and Diverse}

Next, we evaluated whether the IL-4-mediated CD8 upregulation and iCD8SP generation required cell proliferation. In order to track the proliferative history of cells in culture, sorted CD $25^{\text {neg }}$ $\mathrm{TCR} \gamma \delta^{\text {neg }}$ CD4SP thymocytes were labeled with CTV prior to culturing with IL-4, IL-7 or IL-2 (Figure 3A). Both IL-4 and IL-7 induced cell proliferation, resulting in increased cell numbers in the well (mean \pm SD fold change after 7 -day culture: $1,52 \pm 0,70$ vs $2,25 \pm 0,52$ for IL-4 and IL-7, respectively; $n=4)$. Robust proliferation was also observed in all subsets identified upon 7day culture with IL-4, as assessed by both CTV labeling and Ki67 staining (Figure 3B). Nevertheless, we found that a large fraction 


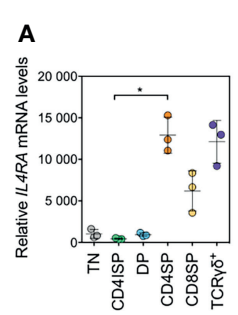

B

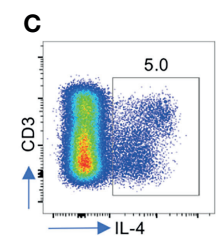

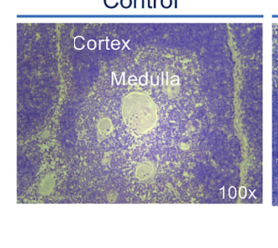

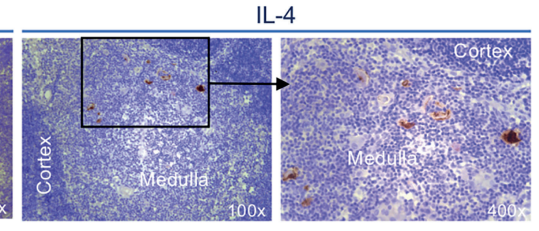

D

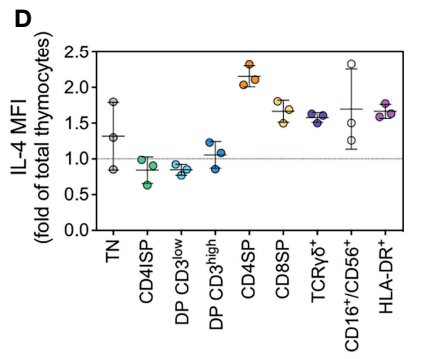

FIGURE 2 | CD4SP thymocytes express IL-4R $\alpha$ and produce high levels of IL-4. (A) IL4RA mRNA levels in developing T cells. TN: triple-negative; CD4ISP: CD4 immature single-positive; DP: double-positive; CD4SP and CD8SP: CD4 ${ }^{+}$or CD8 ${ }^{+}$single-positive. (B) Analysis of IL-4 expression in the human thymus by immunohistochemistry. (C) Flow cytometric analysis of IL-4-producing thymic populations, as frequency of total IL-4 production. (D) Median fluorescence intensity (MFI) of IL-4 ${ }^{+}$cells within each subset analyzed. Results in graphs are presented as mean \pm SD. ${ }^{*} \mathrm{p}<0.05$.

of iCD8SP thymocytes retained high CTV levels and did not express Ki67, and thus did not divide or enter cell cycle (Figure 3B), supporting that IL-4-induced coreceptor reversal of CD4SP thymocytes can occur in the absence of cell division. This observation further excluded the possibility of iDP and iCD8SP cells being solely derived from the proliferation of contaminating $\mathrm{CD}^{+}$cells present at the onset of the culture. Similarly, cell division did not necessarily result in CD8 expression.

We then investigated whether the iCD8SP cells generated from IL-4-treated CD4SP thymocytes (tCD4SP) were stable upon IL-4 removal from the culture. To this end, we sorted IL-4-induced iCD8SP thymocytes, as well as tCD4SP and iDP for comparison, and either replaced IL- 4 by IL- 2 or, as a control, maintained the culture supplemented with IL-4. Whereas the loss of CD8 expression in iDP cells upon IL-4 removal suggested a transient nature of this population, iCD8SP cells preserved their phenotype in the absence of IL-4, indicating that effective coreceptor reversal of CD4SP thymocytes was stable (Figure 3C). Notably, further exposure to IL-4 continued to promote the generation of iCD8SP thymocytes from tCD4SP (Figure 3D). Bcl-2 was upregulated upon IL-4 exposure in all populations, likely promoting cell survival, and the levels attained were similar to those observed upon IL-7 culture (Figure 3E).

Having established the stability of IL-4-induced iCD8SP cells, we asked whether they bared a diverse TCR repertoire. Comparison of the TCR V $\beta$ family profile of iCD8SP thymocytes with ex vivo CD4SP and CD8SP thymocytes revealed that iCD8SP cells indeed present a diverse TCR V $\beta$ repertoire, arguing against their emergence from selective populations within CD4SP thymocytes (Figure 3F).

Overall, we found that IL-4 is able to induce coreceptor reversal in human CD4SP thymocytes in a proliferation- independent manner, generating a stable and diverse population of iCD8SP thymocytes.

\section{IL-4 Induces an Innate-Like Program in Both CD4SP and CD8SP Thymocytes}

Given the central role of IL-4 in the development of innate CD8 T cells we hypothesized that this cytokine could be inducing an innate-like CD8SP phenotype on human CD4SP thymocytes. Indeed, we found an upregulation of Eomes in CD4SP thymocytes cultured with IL-4 in both isolated cells (Figure 4A and Figure S3A) and TOCs (Figure S3B). Our results also showed an increase in the expression of CXCR3, CD44, IFN- $\gamma$, TNF- $\alpha$, perforin and granzyme B, but not of T-bet (Figures $4 \mathrm{~A}-\mathrm{C}$ and Figure S3C).

The innate-like program induced by IL- 4 in CD4SP thymocytes was observed in all the populations generated, independently of CD8 up-regulation (Figures 4A-C and Figure S3A). We further confirmed the lack of correlation between Eomes and CD8 expression in CD4SP cultured with IL-4 at day 7 ( $p=0.102$, Spearman $r=0,77, n=6$ ). Moreover, the innate-like profile induced by IL-4 on CD4SP cells was comparable to that observed in IL-4treated CD8SP thymocytes (Figure 4D and Figure S3D). tSNE clustering of the concatenated flow cytometry data of ex vivo and IL4-exposed CD4SP and CD8SP cells supported the similarity of the populations after IL- 4 treatment, as confirmed by their similar upregulation of Eomes and CXCR3 (Figure 4E). In addition, tSNE analysis supported the absence of a direct association between the expression of CD8 and innate-like markers (Figure 4E). Importantly, this innate-like profile was not observed upon culture with IL-7 (Figure 4F). Moreover, the unique ability of IL4 to induce an innate-like program extended both to CD4 and CD8 $\mathrm{T}$ cells in human cord blood (Figure 4G). 


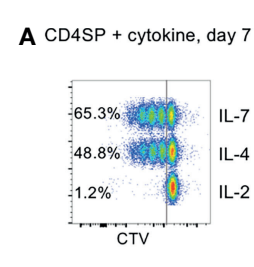

B $\quad \mathrm{CD} 4 \mathrm{SP}+\mathrm{IL}-4$, Day 7

C
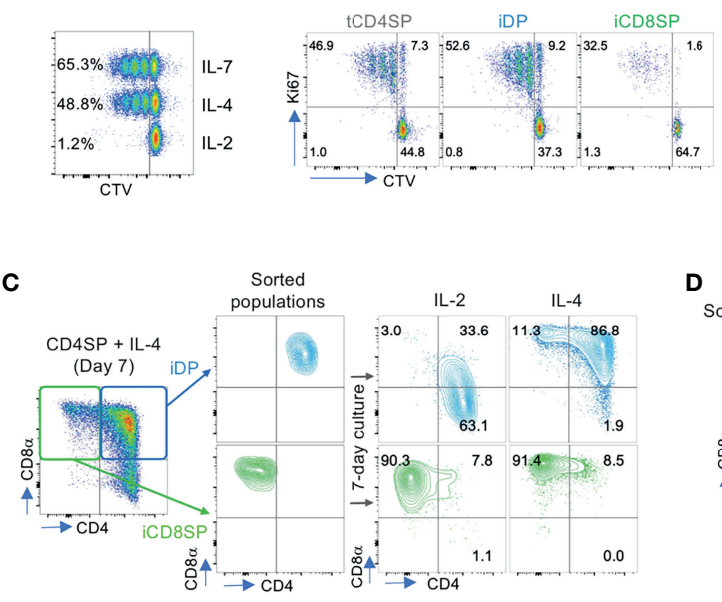

D

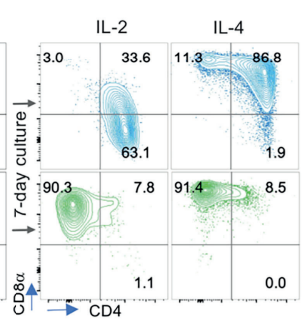

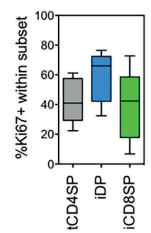

Sorted tCD4SP (day 7)

+7 days of IL- 4

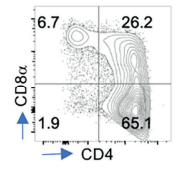

E

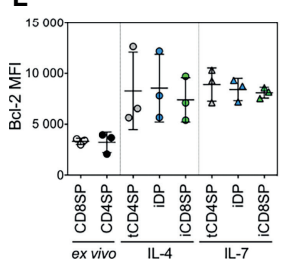

$\mathbf{F}$

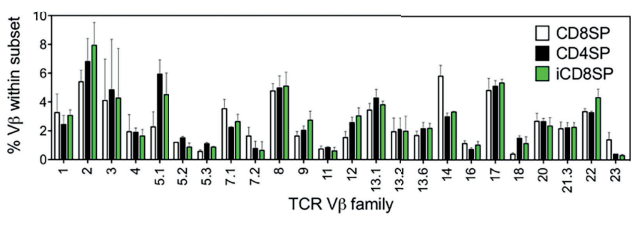

FIGURE 3 | iCD8SP cells generated from CD4SP thymocytes in the presence of IL-4 are stable and diverse. (A) Proliferation of CD4SP thymocytes, sorted as $\mathrm{CD} 3^{\text {high }} \mathrm{CD} 4^{+} \mathrm{CD} 8^{\text {neg }} \mathrm{CD} 25^{\text {neg }} \mathrm{TCR} \gamma \delta^{\text {neg }}$ cells, in response to IL-2, IL-4 and IL-7, as measured by Cell Trace Violet (CTV) dilution. Frequency of CTV ${ }^{\text {neg }}$ cells is presented in each population, and data are representative of 3 experiments. (B) Proliferation of tCD4SP, iDP and iCD8SP thymocytes in response to IL-4, as measured by CTV dilution or Ki67 frequency (graph: $n=5$ ). (C) Stability of IL-4-induced CD8 expression, as assessed by sorting iDP and iCD8SP populations on day 7 of IL-4 culture and either maintaining cells in IL-4 or switching them to IL-2 for 7 more days. (D) CD8 induction in CD4 ${ }^{+}$CD8 ${ }^{\text {neg }}$ (tCD4SP) cells sorted 7 days after IL-4 culture of CD4SP thymocytes and cultured in the presence of IL-4 for 7 additional days. (E) Bcl-2 median fluorescence intensity (MFI) of different populations before and after exposure to IL-4 or IL-7. (F) TCR V $\beta$ repertoire of CD8SP, CD4SP and IL-4-induced iCD8SP thymocytes ( $\mathrm{n}=3$ ). Results in graphs are presented as mean \pm SD.

Overall, our results revealed the potential of IL-4 to induce an innate-like phenotype both in human CD4SP and CD8SP thymocytes, as well as in cord blood $\mathrm{T}$ cells, irrespective of CD8 upregulation. These data thus confirm that the IL-4-induced iCD8SP population includes cells with an innate-like profile.

\section{IL-4 Induction of iCD8SP Cells Is Linked to ThPOK Downregulation}

To characterize the mechanisms by which IL- 4 induces CD8 expression in CD4SP thymocytes, we first determined the mRNA levels of several transcription factors relevant for cell-fate choice, namely ThPOK and GATA3 (CD4 lineage promoting factors), RUNX3, MAZR and ETS1 (CD8 lineage-associated factors), as well as RUNX1 and RUNX2 (described as main players in early stages of human T cell development) $(4,5)$. Analysis of the main thymocyte developmental stages confirmed that ThPOK and RUNX3 levels were highest in CD4SP and CD8SP thymocytes, respectively (Figure 5A). Expression of other RUNX members was increased during early thymocyte development, while Runx3-promoting factor ETS1 was upregulated in mature subsets but did not present a direct association with the CD8 lineage (Figure 5A). CD8SP thymocytes expressed lower GATA3, but higher MAZR levels than CD4SP cells (Figure 5A).
DP and CD4SP thymocytes were further subdivided according to their expression of the positive selection marker CD69 and the maturation marker CD27, respectively, to assess protein expression of ThPOK, Runx3 and Runx1. Our results confirmed the association of ThPOK with the CD4 lineage, of Runx3 with the CD8 lineage, and of Runx1 with early stages of development (Figure 5B and Figure S4A).

Next, we evaluated the levels of transcription factors in IL-4induced populations upon 7-day culture of CD4SP thymocytes both at the transcriptional (Figure 5C) and protein (Figure 5D and Figure S4B) levels. We observed a marked reduction of ThPOK levels in iCD8SP thymocytes, indicating the repression of the CD4 transcriptional program. Notably, iDP thymocytes were similar to tCD4SP thymocytes and did not present the same decrease in ThPOK mRNA levels as iCD8SP, which, together with their loss of iDP phenotype upon IL-4 removal (Figure 3C), further support the transitory nature of the iDP population. We found a minor IL-4-dependent increase in RUNX3 in all the populations that was significant only for tCD4SP, as well as increased RUNX1 levels in iCD8SP cells, although these results were not confirmed at the protein level (Figure 5C and Figure S4B). Finally, we performed unsupervised in-depth clustering analysis of the concatenated flow cytometry data of total 
thymocytes ex vivo and IL-4-exposed CD4SP cells using tSNE dimensional reduction plots (Figure 5D). This data representation further showed that cultured CD4SP thymocytes that downregulated ThPOK clustered closely together with ex vivo CD8SP cells, while Runx3 was less clearly associated with the acquisition of CD8 expression.

Interestingly, we confirmed that the iCD8SP generated in the presence of IL-7 also presented features associated with CD8SP thymocytes, such as CD4 and ThPOK downregulation and increase in CD8 and RUNX3 mRNA levels (Figure S4C), despite the very low frequency of coreceptor reversal observed.

In short, our data demonstrate that IL-4 induces clear downregulation of ThPOK in CD4SP thymocytes, both at the mRNA and protein levels. Given that none of the other transcription factors involved in lineage commitment analyzed was consistently modulated by IL-4, our results suggest that ThPOK downregulation is likely the driver of coreceptor reversal in these cells.
A

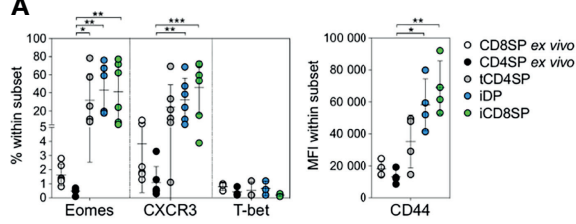

C

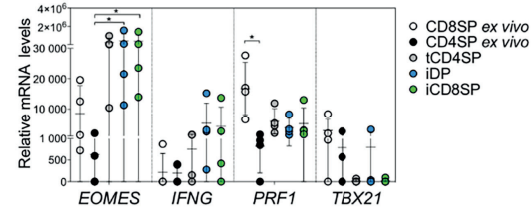

D

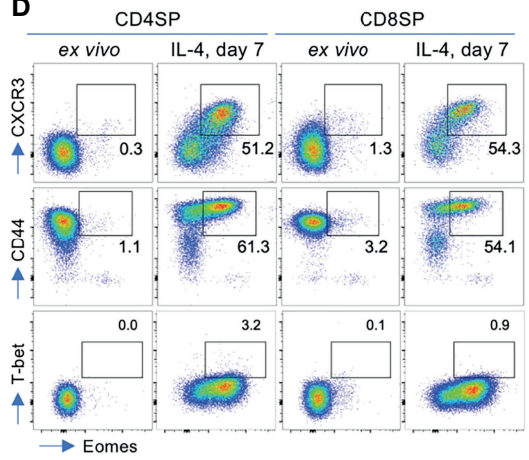

B
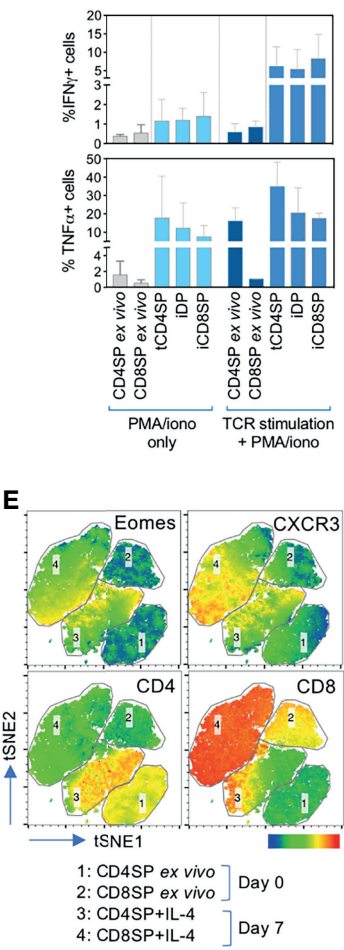

F

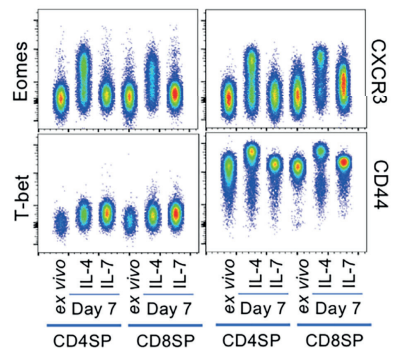

G Cord blood T cells

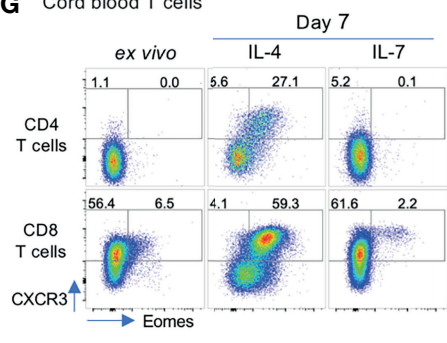

FIGURE 4 | IL-4 induces an innate phenotype on CD4SP thymocytes and cord blood CD4 ${ }^{+}$T cells. (A) Frequency of Eomes, CXCR3 and T-bet and MFI of CD44 within CD4SP or CD8SP thymocytes ex vivo, or tCD4SP, iDP and iCD8SP cells 7 days after exposure to IL-4. (B) Frequency of IFN- $\gamma^{+}$and TNF- $\alpha^{+}$cells within ex vivo CD4SP and CD8SP thymocytes or cells treated with IL-4 upon stimulation with PMA/ionomycin, both with or without TCR stimulation for 5 days ( $\mathrm{n}=3-5$ ). (C) Transcriptional expression of EOMES, IFNG, PRF1 and TBX21 in CD4SP and CD8SP cells ex vivo or after IL-4 culture. (D) Representative dot plots of Eomes, CXCR3, CD44 and T-bet expression in CD4SP and CD8SP thymocytes ex vivo and after 7 days of culture with IL-4. (E) tSNE clustering analysis of the concatenated flow cytometry data of ex vivo and IL-4-exposed CD4SP and CD8SP cells, based on the following markers: CD3, CD4, CD8 $\alpha$, CD8 $\beta$, Eomes and CXCR3. Eomes, CXCR3, CD4 and CD8 expression are presented as heatmap overlays over the populations analyzed. (F) Representative dot plots of innate-related markers in CD4SP and CD8SP thymocytes ex vivo and after 7 days of culture with IL-4 or IL-7. (G) Eomes and CXCR3 expression in cord blood CD4 ${ }^{+}$or CD8 ${ }^{+} \mathrm{T}$ cells ex vivo or cultured with IL-4 or IL-7 for 7 days. Results in graphs are presented as mean $\pm \mathrm{SD} .{ }^{\star} \mathrm{p}<0.05,{ }^{\star \star} \mathrm{p}<0.01,{ }^{\star \star \star} \mathrm{p}<0.001,{ }^{\star \star \star} \mathrm{p}<0.0001$. 
A Human thymocytes ex vivo

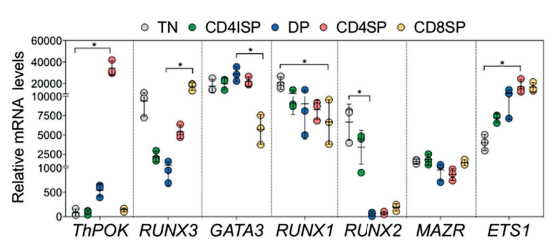

C CD4SP cells + IL-4, 7 days

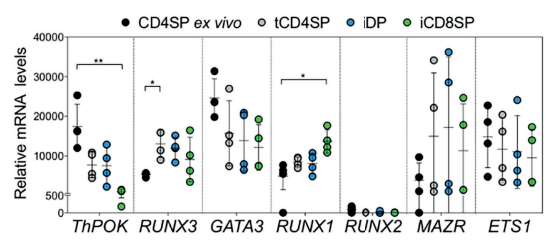

D
B Human thymocytes ex vivo

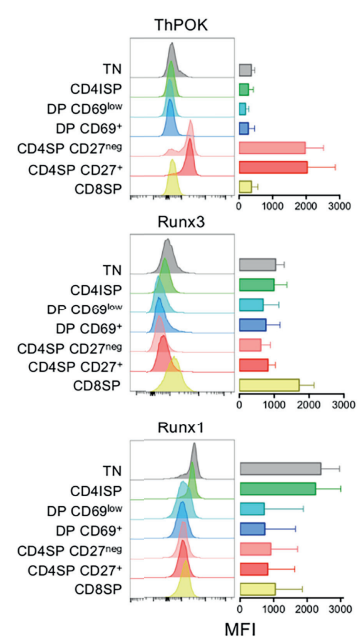

MFI

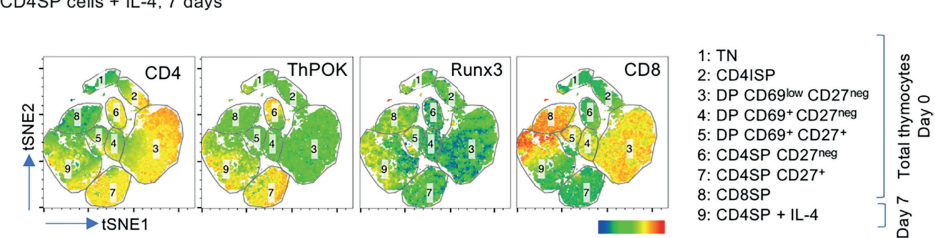

FIGURE 5 | IL-4-induced iCD8SP thymocytes have downregulated ThPOK mRNA and protein. (A) Expression of ThPOK (ZBTB7B), RUNX3, GATA3, RUNX1, RUNX2, MAZR and ETS1 mRNA levels in sorted ex vivo human thymocyte populations. TN: triple-negative; CD4ISP: CD4 immature single-positive; DP: doublepositive; CD4SP and CD8SP: CD4 ${ }^{+}$or CD8 ${ }^{+}$single-positive. (B) Median fluorescence intensity (MFI) of ThPOK, Runx3 and Runx1 expression during human T cell development $(\mathrm{n}=3)$. (C) ThPOK (ZBTB7B), RUNX3, GATA3, RUNX1, RUNX2, MAZR and ETS1 mRNA levels in CD4SP thymocytes ex vivo and in sorted tCD4SP, iDP and iCD8SP populations after IL-4 treatment. (D) tSNE dimensional reduction analysis of concatenated flow cytometry results (total thymocytes and IL-4-treated CD4SP thymocytes), based on the following markers: CD3, CD4, CD8 $\alpha$, CD69, CD27, CD45RA, ThPOK and Runx3. The expression of CD4, ThPOK, Runx3 and $\mathrm{CD} 8$ are presented as overlays over the populations analyzed. Results in graphs are presented as mean $\pm \mathrm{SD}$. ${ }^{*} \mathrm{p}<0.05,{ }^{* *} \mathrm{p}<0.01$.

\section{DISCUSSION}

$\mathrm{CD}^{+}$and $\mathrm{CD}^{+} \mathrm{T}$ cell development occur in the thymus through a sequence of stages that result in the formation of a functional and diverse repertoire. Here, we show that human CD4SP thymocytes have the potential to become CD8SP cells in the presence of IL-4. IL-4-induced iCD8SP thymocytes are stable upon cytokine removal, in agreement with their sustained ThPOK downregulation, and feature a diverse TCR repertoire. Furthermore, IL-4 also induces an innate-like program on CD4SP and CD8SP thymocytes, resulting in the generation of both conventional and Eomes ${ }^{+}$innate-like $\mathrm{CD}^{+} \mathrm{T}$ cells. Importantly, this ability extends to cord blood CD4 T cells.

During human and murine T cell development DP thymocytes reportedly downregulate $\mathrm{CD} 8$ expression, leading to the formation of $\mathrm{CD} 4^{+} \mathrm{CD} 8^{\text {low }}$ cells that can still be converted into the CD8SP lineage upon cytokine signaling $(2,17)$. In mice the main cytokine involved in this kinetic model is IL-7 (32). Although our sorted CD4SP cells may include some of this intermediate CD4SP $\mathrm{CD} 3^{\text {high }}$ cells, it was surprising to find that IL-4 was able to induce much stronger coreceptor reversal in human CD4SP thymocytes than IL-7. In support of a distinct role for IL-4 in human as compared to murine thymocytes, we showed similar ability of IL-4 and IL-7 to increase Bcl-2 levels in CD4SP cells, suggesting comparable effects on cell survival, in contrast to previous murine data (33). Moreover, in an in vitro murine model of positive selection and $\mathrm{CD}^{+} \mathrm{T}$ cell differentiation from $\mathrm{DP}$ thymocytes in the presence of peptide-coated stromal cells and cytokines, culture supplementation with IL-13 resulted in CD8SP formation (34). We were unable to reveal an effect of IL-13 on CD8 expression in CD4SP thymocytes, probably because these cells lack the appropriate receptor, or due to the lack of concomitant exogenous TCR stimulation. The same study also reported the IL-4-induced generation of Eomes ${ }^{+}$innate-like CD8 ${ }^{+}$ thymocytes in their DP-based murine in vitro system, which was not found in cultures supplemented with IL-7, in agreement with our results. Our data support a key role for IL-4 in coreceptor reversal of CD4SP thymocytes, as well as in innate-like $\mathrm{T}$ cell development in the human thymus.

We observed coreceptor reversal not only in CD27 ${ }^{\text {neg }}$ CD4SP cells but also in cells already expressing the maturation and lineage commitment marker CD27, and to lower levels in potentially

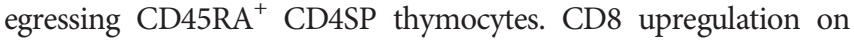
CD4SP thymocytes decreased with maturation stage, though it was not age-dependent in children up to 1 year old, indicating a preserved potential of those cells to generate conventional and/or 
innate CD8SP cells. Importantly, the diverse repertoire and continuous CD8-upregulating potential of the newly formed CD4SP-derived iCD8SP thymocytes strongly supports that IL-4 is able to induce coreceptor reversal in a broad CD4SP population, and not in a specific population or subset.

Our data showed that cord blood CD4 T cells could also be converted into iCD8SP cells in the presence of IL-4. However, peripheral CD4 T cell plasticity and ability to undergo coreceptor reversal decreased with age (children $>$ adult) and differentiation state (naïve $>$ memory). Although we can infer from our data that this is potentially associated with distinct cell-intrinsic properties, additional studies will be required to determine the basis of the differences observed and their relation to the Th2skewed immune response that characterizes neonatal $\mathrm{T}$ cell immunity (35).

IL-4-induced de novo CD8 expression had been described in peripheral CD4 $\mathrm{T}$ cell clones cultured with irradiated allogeneic peripheral blood lymphocytes and JY cells in the presence of phytohemagglutinin, although their phenotype relied on continuous cytokine presence (36). In addition, mature intraepithelial $\mathrm{CD}^{+} \mathrm{T}$ cells in the mouse intestine have been shown to upregulate $\mathrm{CD} 8 \alpha \alpha$ in response to cues such as TGF- $\beta$ and retinoic acid $(14,15)$. However, only $\mathrm{CD} 4^{+} \mathrm{CD} 8^{+}$doublepositive and not $\mathrm{CD} 4^{\text {neg }} \mathrm{CD} 8^{+}$single-positive $\mathrm{T}$ cells were reported to be generated in those studies, and these cells expressed $\mathrm{CD} 8 \alpha \alpha$ and not $\mathrm{CD} 8 \alpha \beta$, which has much stronger affinity for antigen recognition on MHC class I molecules (30). Other $\mathrm{CD}^{+} \mathrm{CD} 8 \alpha \beta^{+}$double-positive $\mathrm{T}$ cells do exist at low frequency in the periphery alongside conventional $\mathrm{CD}^{+}$and $\mathrm{CD}^{+} \mathrm{T}$ cells, and they have been linked to conditions such as infections, autoimmune diseases and cancer (37). In cancer settings, $\mathrm{CD} 4^{+} \mathrm{CD} 8^{+}$cells were shown to produce IL-4 themselves (37), thus probably promoting the induction of IL4-related programs. They appear to play an important role at peripheral sites, and some studies have reported their cytotoxic potential, though they may also have suppressive function (37). Whether peripheral $\mathrm{CD} 4{ }^{+} \mathrm{CD} 8 \alpha \beta^{+}$double-positive $\mathrm{T}$ cells include a transient population giving rise to $\mathrm{CD}^{+} \mathrm{T}$ cells in the presence of IL-4 remains to be determined.

A question that arises from our results is how TCR specificity impacts on CD4-derived CD8 T cells. Antigen-restricted MHC class II-specific $\mathrm{CD}^{+} \mathrm{T}$ cells have been described in HIVinfected patients (38-40). Moreover, a recent study reports the in vivo generation of a $\mathrm{CD} 4-\mathrm{CD} 8 \alpha \beta^{+} \mathrm{MHC}$ II-recognizing lineage from effector $\mathrm{CD}^{+} \mathrm{T}$ cells using murine models of acute infection (41). In addition, an increase in thymic innate CD8 thymocytes has recently been shown to be associated with T. cruzi murine infection in vivo, and those cells were able to protect against lethal infection in an antigen-independent manner (42). In line with these reports, IL-4-induced iCD8SP cells may support an MHC-II-biased or even an MHC-unbiased response to foreign pathogens.

In the thymus, IL- 4 has been described to be mainly produced by NKT cells or T-T CD4 thymocytes $(27,43)$. We directly quantified IL-4 production ex vivo in human postnatal thymocytes in the absence of exogenous stimulation and identified, on a per cell basis, CD4SP thymocytes as the strongest IL-4 producers. We also found that a large fraction of low-level IL-4-producing cells are DP CD $3^{\text {low }}$ thymocytes, in agreement with a previous report of measurable IL4 mRNA levels in this population in fetal thymuses (44). Importantly, our histological data showed that IL-4 was mainly expressed in the medullary region in postnatal thymuses, in support of its physiological role in CD4SP thymocyte plasticity. The high IL4 production by postnatal human CD4SP thymocytes and their elevated IL-4R $\alpha$ levels argue in favor of a possible feedback loop that can result in a shift of their fate. In agreement, we found an inversion of the CD4/CD8 ratio in thymic tissue supplemented with IL-4, indicating that IL-4 may impact T cell development in situations where IL-4 is systemically increased, such as chronic inflammation (45).

IL-4-mediated conversion of CD4SP thymocytes into stable iCD8SP cells was paralleled by transcriptional and translational downregulation of ThPOK, the master transcription factor for $\mathrm{CD}^{+} \mathrm{T}$ cell commitment, which likely constituted the molecular basis for the observed coreceptor reversal. In support of this possibility, ThPOK was shown to directly promote CD8 silencing during murine CD4 lineage commitment, and to suppress CD8 lineage gene expression in peripheral $\mathrm{CD}^{+} \mathrm{T}$ cells (13). Moreover, disruption of ThPOK or mutations in its zinc finger domains redirect MHC class II-restricted murine thymocytes into the $\mathrm{CD}^{+}$lineage, leading to the formation of MHC class IIspecific CD8SP cells $(6,7,46-48)$. In addition, post-thymic loss of ThPOK derepresses the CTL program in mature CD4 T cells in mouse blood and peripheral tissues under inflammatory conditions (14). Since ThPOK also negatively regulates RUNX3 expression, a crucial factor for full commitment to the cytotoxic CD8 T cell lineage, it could be expected that IL-4 would induce an increase in RUNX3 expression concomitantly with the ThPOK downregulation (46). However, we did not find substantial differences in RUNX3 mRNA or protein levels upon culture with IL-4. Importantly, we noticed that ex vivo CD4SP thymocytes already expressed RUNX3, while ex vivo CD8SP thymocytes completely lacked ThPOK expression. This was in agreement with previous whole-genome sequencing data of human thymocytes, where ThPOK mRNA was only detected in CD4SP while RUNX3 mRNA was present both in CD8SP and CD4SP cells (49). We thus hypothesize that IL-4-induced ThPOK downregulation on CD4SP cells was enough to revert their fate and induce a cytotoxic program due to the basal expression of RUNX3 by CD4SP thymocytes, which may guarantee an epigenetic "poised" state that allows coreceptor reversal of this population. The mechanisms by which IL-4 induces ThPOK downregulation and CD8 expression in CD4SP thymocytes, including STAT6-dependent and independent signaling pathways and epigenetic regulation, are currently under investigation.

IL-4 has been described to promote Eomes expression on $\mathrm{CD}^{+} \mathrm{T}$ cells and to be required for its maintenance in thymic innate-like and peripheral memory $\mathrm{CD}^{+} \mathrm{T}$ cells in mice $(22,27$, $50)$. We show here that IL-4 induces not only the formation of conventional CD8SP thymocytes from human postnatal CD4SP 
thymocytes, but also the emergence of Eomes ${ }^{+}$T-bet ${ }^{\text {neg }}$ innatelike iCD8SP thymocytes, which are very similar to those derived from paired CD8SP thymocytes since they also express high levels of innate-associated markers, such as CXCR3 and CD44. We further extended these results to the periphery, as cord blood T cells are still able to generate Eomes ${ }^{+}$cells in response to IL-4, with potential implications for early defense mechanisms in the neonatal period.

In conclusion, we show here that IL- 4 modulates the plasticity of human CD4SP thymocytes and cord blood $\mathrm{CD}^{+} \mathrm{T}$ cells, promoting their conversion into $\mathrm{CD}^{+} \mathrm{T}$ cells, as well as the acquisition of an innate-like profile. This may be particularly important during early childhood, when a fully competent adaptive immune response is yet to be established, and points to novel pathways to modulate anti-viral immunity and tumor immune responses.

\section{DATA AVAILABILITY STATEMENT}

The original contributions presented in the study are included in the article/Supplementary Material. The raw data supporting the conclusions of this article will be made available by the authors. Further inquiries can be directed to the corresponding authors.

\section{ETHICS STATEMENT}

The study was reviewed and approved by the Ethical Boards of Hospital de Santa Cruz, Faculty of Medicine of the University of Lisbon and CHULN Lisbon, Portugal. Written informed consent to participate in this study was provided by the participants' legal guardian/next of kin.

\section{REFERENCES}

1. Spits H. Development of $\alpha \beta$ T Cells in the Human Thymus. Nat Rev Immunol (2002) 2:760-72. doi: 10.1038/nri913

2. Vanhecke D, Verhasselt B, Smedt MD, Leclercq G, Plum J, Vandekerckhove B. Human Thymocytes Become Lineage Committed at an Early Postselection CD69+ Stage, Before the Onset of Functional Maturation. J Immunol (1997) 159:5973-83.

3. Vanhecke D, Leclercq G, Plum J, Vandekerckhove B. Characterization of Distinct Stages During the Differentiation of Human CD69+CD3+ Thymocytes and Identification of Thymic Emigrants. J Immunol (1995) 155:1862-72

4. Egawa T. Regulation of CD4 and CD8 Coreceptor Expression and CD4 Versus CD8 Lineage Decisions. Adv Immunol (2015) 125:1-40. doi: 10.1016/ bs.ai.2014.09.001

5. Taniuchi I. CD4 Helper and CD8 Cytotoxic T Cell Differentiation. Annu Rev Immunol (2018) 36:579-601. doi: 10.1146/annurev-immunol-042617053411

6. He X, He X, Dave VP, Zhang Y, Hua X, Nicolas E, et al. The Zinc Finger Transcription Factor Th-POK Regulates CD4 Versus CD8 TCell Lineage Commitment. Nature (2005) 433:826-33. doi: 10.1038/ nature 03338

7. Sun G, Liu X, Mercado P, Jenkinson SR, Kypriotou M, Feigenbaum L, et al. The Zinc Finger Protein Ckrox Directs CD4 Lineage Differentiation During

\section{AUTHOR CONTRIBUTIONS}

HN-C and AS designed the study. HN-C, AR-d-S, AP, and LM performed research and analyzed the data. $\mathrm{HN}-\mathrm{C}, \mathrm{JB}$, and AS discussed the results. HN-C and AS wrote the paper. All authors contributed to the article and approved the submitted version.

\section{FUNDING}

This work received funding from PAC - PRECISE - LISBOA-010145-FEDER-016394, co-funded by FEDER through POR Lisboa 2020 - Programa Operacional Regional de Lisboa PORTUGAL 2020 and Fundação para a Ciência e a Tecnologia (FCT). HN-C and LM received fellowships from FCT.

\section{ACKNOWLEDGMENTS}

We sincerely thank the patients and families; Miguel Abecasis and Rui Anjos for human thymus sample collection; Helena Ferreira for cord blood collection; Francisca Matos and nurses and staff at the Hospital de Santa Cruz and at ENT/CHULN for technical assistance; the team members of the Flow Cytometry, Imaging and Histology Facilites at iMM-JLA; Bruno SilvaSantos, Marc Veldhoen and Miguel Prudêncio for critical reading of the manuscript.

\section{SUPPLEMENTARY MATERIAL}

The Supplementary Material for this article can be found online at: https://www.frontiersin.org/articles/10.3389/fimmu.2022. 834033/full\#supplementary-material

Intrathymic T Cell Positive Selection. Nat Immunol (2005) 6:373-81. doi $10.1038 /$ nil 183

8. Taniuchi I, Osato M, Egawa T, Sunshine MJ, Bae S-C, Komori T, et al. Differential Requirements for Runx Proteins in CD4 Repression and Epigenetic Silencing During T Lymphocyte Development. Cell (2002) 111:621-33. doi: 10.1016/S0092-8674(02)01111-X

9. Woolf E, Xiao C, Fainaru O, Lotem J, Rosen D, Negreanu V, et al. Runx3 and Runx1 are Required for CD8 T Cell Development During Thymopoiesis. Proc Natl Acad Sci (2003) 100:7731-6. doi: 10.1073/ pnas. 1232420100

10. Hernández-Hoyos G, Anderson MK, Wang C, Rothenberg EV, Alberola-Ila J. GATA-3 Expression is Controlled by TCR Signals and Regulates CD4/CD8 Differentiation. Immunity (2003) 19:83-94. doi: 10.1016/S1074-7613(03) 00176-6

11. Pai S-Y, Truitt ML, Ting C-N, Leiden JM, Glimcher LH, Ho I-C. Critical Roles for Transcription Factor GATA-3 in Thymocyte Development. Immunity (2003) 19:863-75. doi: 10.1016/S1074-7613(03)00328-5

12. Sakaguchi S, Hombauer M, Bilic I, Naoe Y, Schebesta A, Taniuchi I, et al. The Zinc-Finger Protein MAZR is Part of the Transcription Factor Network That Controls the CD4 Versus CD8 Lineage Fate of Double-Positive Thymocytes. Nat Immunol (2010) 11:442-8. doi: 10.1038/ni.1860

13. Wang L, Wildt KF, Castro E, Xiong Y, Feigenbaum L, Tessarollo L, et al. The Zinc Finger Transcription Factor Zbtb7b Represses CD8-Lineage Gene 
Expression in Peripheral CD4+ T Cells. Immunity (2008) 29:876-87. doi: 10.1016/j.immuni.2008.09.019

14. Mucida D, Husain MM, Muroi S, van Wijk F, Shinnakasu R, Naoe Y, et al. Transcriptional Reprogramming of Mature CD4+ Helper T Cells Generates Distinct MHC Class II-restricted Cytotoxic T Lymphocytes. Nat Immunol (2013) 14:281-9. doi: 10.1038/ni.2523

15. Reis B, Rogoz A, Costa-Pinto F, Mucida D. Mutual Expression of Runx 3 and ThPOK Regulates Intestinal CD4 T Cell Immunity: P-172 YI. Inflamm Bowel Dis (2012) 18:S83-4. doi: 10.1097/00054725-201212001-00205

16. Park J-H, Adoro S, Guinter T, Erman B, Alag AS, Catalfamo M, et al. Signaling by Intrathymic Cytokines, Not T Cell Antigen Receptors, Specifies CD8 Lineage Choice and Promotes the Differentiation of Cytotoxic-Lineage $\mathrm{T}$ Cells. Nat Immunol (2010) 11:257-64. doi: 10.1038/ni.1840

17. Brugnera E, Bhandoola A, Cibotti R, Yu Q, Guinter TI, Yamashita Y, et al. Coreceptor Reversal in the Thymus: Signaled CD4+8+ Thymocytes Initially Terminate CD8 Transcription Even When Differentiating Into CD8+ T Cells. Immunity (2000) 13:59-71. doi: 10.1016/S1074-7613(00)00008-X

18. Silva-Filho JL, Caruso-Neves C, Pinheiro AAS. IL-4: An Important Cytokine in Determining the Fate of T Cells. Biophys Rev (2014) 6:111-8. doi: 10.1007/ s12551-013-0133-z

19. Luzina IG, Keegan AD, Heller NM, Rook GAW, Shea-Donohue T, Atamas SP. Regulation of Inflammation by Interleukin-4: A Review of "Alternatives." J Leukoc Biol (2012) 92:753-64. doi: 10.1189/jlb.0412214

20. Carvalho LH, Sano G-I, Hafalla JCR, Morrot A, Curotto de Lafaille MA, Zavala F. IL-4-Secreting CD4+ T Cells are Crucial to the Development of CD8+ T-Cell Responses Against Malaria Liver Stages. Nat Med (2002) 8:166-70. doi: 10.1038/ nm0202-166

21. Morris SC, Heidorn SM, Herbert DR, Perkins C, Hildeman DA, Khodoun MV, et al. Endogenously Produced IL-4 Nonredundantly Stimulates CD8+ T Cell Proliferation. J Immunol (2009) 182:1429-38. doi: 10.4049/jimmunol.182.3.1429

22. Oliver JA, Stolberg VR, Chensue SW, King PD. IL-4 Acts as a Potent Stimulator of IFN- $\gamma$ Expression in CD8 + T Cells Through STAT6Dependent and Independent Induction of Eomesodermin and T-Bet. Cytokine (2012) 57:191-9. doi: 10.1016/j.cyto.2011.10.006

23. Wijesundara DK, Tscharke DC, Jackson RJ, Ranasinghe C. Reduced Interleukin-4 Receptor $\alpha$ Expression on CD8+ T Cells Correlates With Higher Quality Anti-Viral Immunity. PloS One (2013) 8:e55788. doi: 10.1371/journal.pone.0055788

24. Jameson SC, Lee YJ, Hogquist KA. Innate Memory T Cells. Adv Immunol (2015) 126:173-213. doi: 10.1016/bs.ai.2014.12.001

25. White JT, Cross EW, Kedl RM. Antigen-Inexperienced Memory CD8+ T Cells: Where They Come From and Why We Need Them. Nat Rev Immunol (2017) 17:391-400. doi: 10.1038/nri.2017.34

26. Jacomet F, Cayssials E, Basbous S, Levescot A, Piccirilli N, Desmier D, et al. Evidence for Eomesodermin-Expressing Innate-Like CD8 ${ }^{+}$KIR/NKG2A ${ }^{+} \mathrm{T}$ Cells in Human Adults and Cord Blood Samples: Innate Immunity. Eur $J$ Immunol (2015) 45:1926-33. doi: 10.1002/eji.201545539

27. Min HS, Lee YJ, Jeon YK, Kim EJ, Kang BH, Jung KC, et al. MHC Class IIRestricted Interaction Between Thymocytes Plays an Essential Role in the Production of Innate CD8 ${ }^{+}$T Cells. J Immunol (2011) 186:5749-57. doi: 10.4049/jimmunol.1002825

28. Renkema KR, Lee J-Y, Lee YJ, Hamilton SE, Hogquist KA, Jameson SC. IL-4 Sensitivity Shapes the Peripheral CD8+ T Cell Pool and Response to Infection. J Exp Med (2016) 213:1319-29. doi: 10.1084/jem.20151359

29. Nunes-Cabaço H, Matoso P, Foxall RB, Tendeiro R, Pires AR, Carvalho T, et al. Thymic HIV-2 Infection Uncovers Posttranscriptional Control of Viral Replication in Human Thymocytes. J Virol (2015) 89:2201-8. doi: 10.1128/JVI.03047-14

30. Bosselut R, Kubo S, Guinter T, Kopacz JL, Altman JD, Feigenbaum L, et al. Role of CD8beta Domains in CD8 Coreceptor Function: Importance for MHC I Binding, Signaling, and Positive Selection of CD8+ T Cells in the Thymus. Immunity (2000) 12:409-18. doi: 10.1016/S1074-7613(00)80193-4

31. Nakayama K, Nakayama K, Negishi I, Kuida K, Louie MC, Kanagawa O, et al. Requirement for CD8 Beta Chain in Positive Selection of CD8-Lineage T Cells. Science (1994) 263:1131-3. doi: 10.1126/science.8108731

32. McCaughtry TM, Etzensperger R, Alag A, Tai X, Kurtulus S, Park J-H, et al. Conditional Deletion of Cytokine Receptor Chains Reveals That IL-7 and IL15 Specify CD8 Cytotoxic Lineage Fate in the Thymus. J Exp Med (2012) 209:2263-76. doi: 10.1084/jem.20121505
33. Yu Q, Erman B, Bhandoola A, Sharrow SO, Singer A. In Vitro Evidence That Cytokine Receptor Signals Are Required for Differentiation of Double Positive Thymocytes Into Functionally Mature CD8 ${ }^{+}$T Cells. J Exp Med (2003) 197:475-87. doi: 10.1084/jem.20021765

34. Rafei M, Rouette A, Brochu S, Vanegas JR, Perreault C. Differential Effects of C Cytokines on Postselection Differentiation of CD8 Thymocytes. Blood (2013) 121:107-17. doi: 10.1182/blood-2012-05-433508

35. Debock I, Flamand V. Unbalanced Neonatal CD4+ T-Cell Immunity. Front Immunol (2014) 5:393. doi: 10.3389/fimmu.2014.00393

36. Paliard X, Malefijt RW, de Vries JE, Spits H. Interleukin-4 Mediates CD8 Induction on Human CD4+ T-Cell Clones. Nature (1988) 335:642-4. doi: $10.1038 / 335642 \mathrm{a} 0$

37. Overgaard NH, Jung J-W, Steptoe RJ, Wells JW. CD4 ${ }^{+} / \mathrm{CD} 8{ }^{+}$DoublePositive T Cells: More Than Just a Developmental Stage? J Leukoc Biol (2015) 97:31-8. doi: 10.1189/jlb.1RU0814-382

38. Migueles SA, Connors M. Class II-Restricted CD8s: New Lessons Violate Old Paradigms. Immunity (2016) 45:712-4. doi: 10.1016/j.immuni.2016.10.004

39. Nyanhete TE, Frisbee AL, Bradley T, Faison WJ, Robins E, Payne T, et al. HLA Class II-Restricted CD8+ T Cells in HIV-1 Virus Controllers. Sci Rep (2019) 9:10165. doi: 10.1038/s41598-019-46462-8

40. Ranasinghe S, Lamothe PA, Soghoian DZ, Kazer SW, Cole MB, Shalek AK, et al. Antiviral CD8 + T Cells Restricted by Human Leukocyte Antigen Class II Exist During Natural HIV Infection and Exhibit Clonal Expansion. Immunity (2016) 45:917-30. doi: 10.1016/j.immuni.2016.09.015

41. Robins E, Zheng M, Ni Q, Liu S, Liang C, Zhang B, et al. Conversion of Effector CD4+ T Cells to a CD8+ MHC II-Recognizing Lineage. Cell Mol Immunol (2021) 18:150-61. doi: 10.1038/s41423-019-0347-5

42. Baez NS, Cerbán F, Savid-Frontera C, Hodge DL, Tosello J, Acosta-Rodriguez E, et al. Thymic Expression of IL-4 and IL-15 After Systemic Inflammatory or Infectious Th1 Disease Processes Induce the Acquisition of "Innate" Characteristics During CD8+ T Cell Development. PloS Pathog (2019) 15: e1007456. doi: 10.1371/journal.ppat.1007456

43. Lee YJ, Jameson SC, Hogquist KA. Alternative Memory in the CD8 T Cell Lineage. Trends Immunol (2011) 32:50-6. doi: 10.1016/j.it.2010.12.004

44. Vandekerckhove BAE, Bircena A, Schols D, Mohan-Peterson S, Spits H, Roncarolo M-C. In Vivo Cytokine Expression in the Thymus. CD3high Human Thymocytes Are Activated and Already Functionally Differentiated in Helper and Cytotoxic Cells. J Immunol (1994) 152:1738-43.

45. Nappo G, Handle F, Santer FR, McNeill RV, Seed RI, Collins AT, et al. The Immunosuppressive Cytokine Interleukin-4 Increases the Clonogenic Potential of Prostate Stem-Like Cells by Activation of STAT6 Signalling. Oncogenesis (2017) 6:e342-2. doi: 10.1038/oncsis.2017.23

46. Egawa T, Littman DR. The Transcription Factor ThPOK Acts Late in Helper T Cell Lineage Specification and Suppresses Runx-Mediated Commitment to the Cytotoxic T Cell Lineage. Nat Immunol (2008) 9:1131-9. doi: 10.1038/ni.1652

47. Keefe R, Dave V, Allman D, Wiest D, Kappes DJ. Regulation of Lineage Commitment Distinct From Positive Selection. Science (1999) 286:1149-53. doi: 10.1126/science.286.5442.1149

48. Vacchio MS, Bosselut R. What Happens in the Thymus Does Not Stay in the Thymus: How T Cells Recycle the CD $4{ }^{+}-\mathrm{CD} 8{ }^{+}$Lineage Commitment Transcriptional Circuitry To Control Their Function. J Immunol (2016) 196:4848-56. doi: 10.4049/jimmunol.1600415

49. Rodriguez RM, Suarez-Alvarez B, Mosén-Ansorena D, García-Peydró M, Fuentes P, García-León MJ, et al. Regulation of the Transcriptional Program by DNA Methylation During Human $\alpha \beta$ T-Cell Development. Nucleic Acids Res (2015) 43:760-74. doi: 10.1093/nar/gku1340

50. Takemoto N, Intlekofer AM, Northrup JT, Wherry EJ, Reiner SL. Cutting Edge: IL-12 Inversely Regulates T-Bet and Eomesodermin Expression During Pathogen-Induced CD8 ${ }^{+}$T Cell Differentiation. J Immunol (2006) 177:75159. doi: 10.4049/jimmunol.177.11.7515

Conflict of Interest: The authors declare that the research was conducted in the absence of any commercial or financial relationships that could be construed as a potential conflict of interest.

Publisher's Note: All claims expressed in this article are solely those of the authors and do not necessarily represent those of their affiliated organizations, or those of the publisher, the editors and the reviewers. Any product that may be evaluated in 
this article, or claim that may be made by its manufacturer, is not guaranteed or endorsed by the publisher.

Copyright (c) 2022 Nunes-Cabaço, Ramalho-dos-Santos, Pires, Martins, Barata and Sousa. This is an open-access article distributed under the terms of the Creative
Commons Attribution License (CC BY). The use, distribution or reproduction in other forums is permitted, provided the original author(s) and the copyright owner(s) are credited and that the original publication in this journal is cited, in accordance with accepted academic practice. No use, distribution or reproduction is permitted which does not comply with these terms. 